\title{
Investigation of the asperity point load mechanism for thermal elastohydrodynamic conditions
}

\author{
Carl-Magnus Everitt ${ }^{1,1}$, and Bo Alfredsson ${ }^{1}$ \\ ${ }^{1}$ Department of Solid Mechanics, KTH - Royal Institute of Technology
}

\begin{abstract}
The rolling contact fatigue damage called pitting or spalling develops more frequently in surfaces with negative than positive slip. Since normal line loads do not cause any tensile surface stresses this investigation considers the effects of small point shaped asperities. Shear traction causes tensile stresses at the trailing edge of asperities entering the contact at negative slip. At positive slip the tensile stresses appear at the leading edge when the asperities exit the contact. It was found that the trailing edge of the asperity breaks through the lubrication film at contact entry. This causes negative slip to be more detrimental than positive slip. At negative slip the location of large frictional shear stresses and tension stresses from normal asperity contact coincide.
\end{abstract}

\section{Introduction}

Highly loaded gear and bearing surfaces will eventually fail due to rolling contact fatigue (RCF). One form of RCF is pitting, also called spalling, where the complete damage is the result of fatigue crack growth until the detachment of a piece of surface material. The damage can initiate at the surface or below it. This work analyses one initiation mechanism of the surface initiated cracks. The surface cavity often has the characteristic sea-shell shape presented in Fig. 1a. It is established [1] that the damage almost exclusively develops where friction acts against the rolling direction (negative slip). The process starts with fatigue initiation at the tip of the sea-shell shaped pit. It then slowly grows in the forward rolling direction [1]. The damage driving mechanism is however still debated.

The asperity point load mechanism for RCF resides in surface peaks creating point contacts which are surrounded by a tensile surface stress. Inside the contact, the nominal pressure ensures that the surface stresses remain compressive. However, on the outside of the contact entering or exiting asperity the tensile stress may be high enough to cause crack initiation and growth, see Fig. 1b. Experiments show that the crack propagation profile from a point load follows the characteristic cross-sectional profile of pits [2]. Simulations, based on rolling contacts with asperities and a mode I crack direction criterion, shows that the full profile agrees with the sea-shell pit shape [3]. In the current study several thermal 
elastohydrodynamic (TEHL) simulations were performed to investigate the effect of sliding at varying the slide to roll ratio, SRR. Surface fatigue was evaluated through the Findley criterion. The goal was to show that the asperity point load mechanism can explains why pits initiates more often for negative than positive slip.
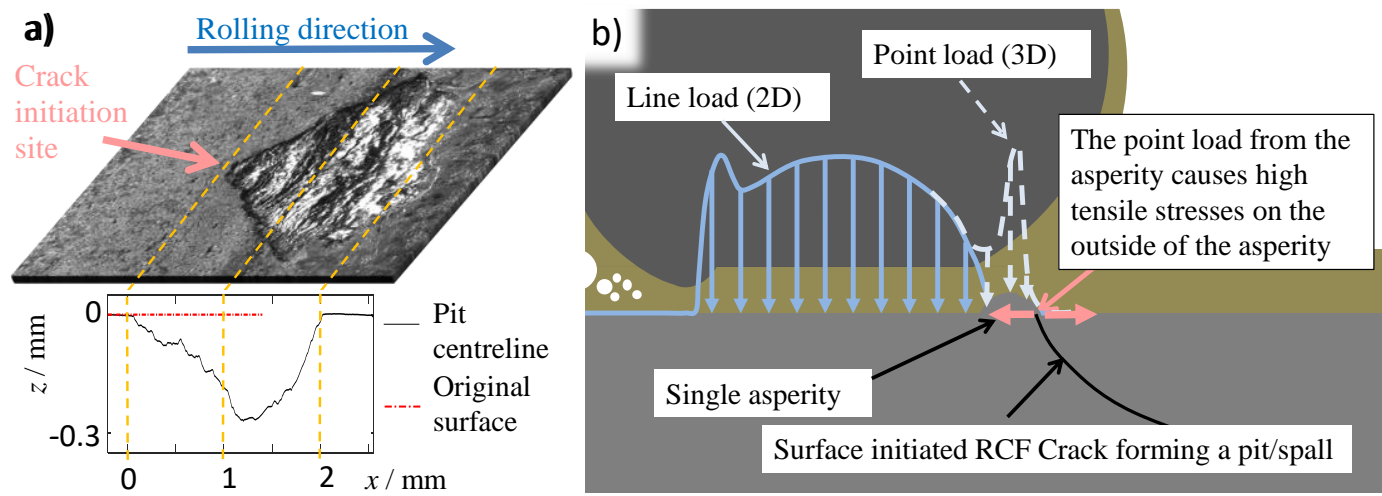

Fig. 1 a) A tilted surface view of a RCF pit together with its cross-sectional profile. b) Schematic view of the asperity point load mechanism inducing a surface initiated RCF crack at asperity entry.

\section{Theoretical background}

The rolling contact was modelled fully flooded with Reynolds' equation for thin films:

$$
\frac{\partial}{\partial x}\left(\frac{\rho h^{3}}{12 \eta} \frac{\partial}{\partial x} p\right)+\frac{\partial}{\partial y}\left(\frac{\rho h^{3}}{12 \eta} \frac{\partial}{\partial y} p\right)-u_{\mathrm{m}} \frac{\partial}{\partial x}(\rho h)-\frac{\partial}{\partial t}(\rho h)=0
$$

In Eq. (1) $p$ is the pressure, $h$ is the local film thickness, $u_{\mathrm{m}}$ is the mean entrainment velocity, $\rho$ is the density and $\eta$ is the viscosity. Since the width of the contact was larger than $1000 \cdot h$, average values were used in the thickness direction. Asperity effects were captured by solving the differential equation in both the rolling direction (RD) and the transverse direction (TD). Cavitation was treated by forcing $p \geq 0$. The asperity shape was modelled with an axisymmetric cosine profile; see Everitt and Alfredsson [4]. The pressure-viscosity relation of the lubricant was described by Roelands' equation:

$$
\eta=\eta_{0}(\Gamma) \exp \left(\left[\ln \left(\eta_{0}(\Gamma)\right)+9.67\right]\left[-1+\left(1+5.1 \cdot 10^{-9} p\right)^{Z_{\mathrm{R}}(\Gamma)}\right]\right)
$$

Equations for $Z_{\mathrm{R}}, \eta_{0}$ and $\rho$ along with material parameters were collected from Larsson $e t$ al. [5]. The shear limit proposed by Bair et al. [6] was used to limit the shear tractions:

$$
\tau_{1}=\tau_{0}+\gamma p
$$

where $\gamma$ is the limiting shear stress coefficient and $\tau_{0}$ is the shear limit at $p=0$. Coulomb friction was used for metal contact, with the friction coefficient $\mu_{\text {Dry }}=0.3$. 


\subsection{Thermal model}

The energy dissipation per lubricant volume was estimated by integrating the shear stresses times the shear rates and dividing by the film thickness. It was combined with compressibility and the assumption of a constant temperature through the fluid thickness. To incorporate these effects the incremental change of energy density was formulated as

$$
\begin{aligned}
\frac{d\left(\rho C_{\mathrm{p}} \Gamma\right)}{d t}= & \frac{h^{2}(\nabla p)^{2}}{12 \eta}+\left|\frac{\mathbf{u}_{\mathrm{s}} \cdot \nabla p}{2}\right|+\eta \frac{\mathbf{u}_{\mathrm{s}} \cdot \mathbf{u}_{\mathrm{s}}}{h^{2}}-\frac{\Gamma}{\rho} \frac{\partial \rho}{\partial \Gamma}\left(\mathbf{u}_{\mathrm{a}} \nabla p+\frac{\partial p}{\partial t}\right) \\
& -\rho C_{\mathrm{p}} \mathbf{u}_{\mathrm{a}} \cdot \nabla \Gamma+\nabla \cdot \kappa \nabla \Gamma
\end{aligned}
$$

where $\mathbf{u}_{\mathrm{a}}=u_{\mathrm{m}}-h^{2} \nabla p / 12 \eta$ is the average fluid velocity and $\mathbf{u}_{\mathrm{s}}$ is the sliding velocity; see for example by Cheng and Sternlicht [7] for a similar formulation. The energy dissipation through the solids were modelled with the same equation except that the energy source terms, the first four terms on the right hand side of Eq. (4) were excluded since the solids were elastic without internal heat generation. Any effects on the lubricant film from thermal expansion of the solids were omitted.

The power generated by sliding metal contacts was added to the surface nodes of the metals instead of the lubricant. The power was then equally distributed between the two surfaces. The metal contact had a high capacity of heat conduction since there was no isolating lubricant present.

\subsection{Fatigue evaluation}

Repeated contact pressure and traction gave rise to cyclic stresses in the solids. Fatigue was evaluated using the Findley criterion. It was selected based on earlier studies on cases with large compressive mean stresses in combination with tensile in-surface stresses $[8,9]$. In dimensionless form, the Findley criterion is

$$
F i=\frac{\left(\tau_{\mathrm{amp}}+\kappa_{\mathrm{F}} \sigma_{n, \max }\right)_{\max }}{\sigma_{\mathrm{eF}}}
$$

where $\tau_{\mathrm{amp}}$ is the shear stress amplitude on a plane and $\sigma_{\mathrm{n}, \max }$ is the maximum normal stress on the same plane. $\kappa_{\mathrm{F}}=0.627$ and $\sigma_{\mathrm{eF}}=625 \mathrm{MPa}$ are the normal stress parameter and the endurance limit for the current case carburized gear steel [4]. The plane that maximizes $\mathrm{Fi}$ is searched for. An index value above unity predicts fatigue damage.

\section{Numerical setup}

The numerical program was built on Huang's code [10]. It starts by solving a timeindependent case of a smooth cylinder rolling on a plane. To minimize the time spent to obtain the steady-state solution the simulations were first roughly solved for a course mesh. Then the mesh was refined in three steps. At the approximate convergence of $P, \Gamma$ and $H_{0}$ for a coarser mesh, the solution was transferred to a refined mesh. Each refinement doubled the resolution in all directions. The final mesh contained $257 \cdot 49$ nodes in the lubricant. When the time-independent model had converged, the transient problem was introduced and the asperity travelled through the model in 257 time steps. In order to get a fully 
coupled solution both $P$ and $\Gamma$ were updated in each iteration of each time step. To stabilize the solution the relaxation parameters in the Gauss-Seidel and the Jacobi iteration methods were adjusted based on the residual. The temperature field were updated with a newton forward iteration scheme. To enable a stable temperature solution sub time steps where introduced inside the temperature routine. The global structure of the program is shown in Fig. 2. A more detailed description has been provided by Everitt and Alfredsson in their article from 2019 [11].

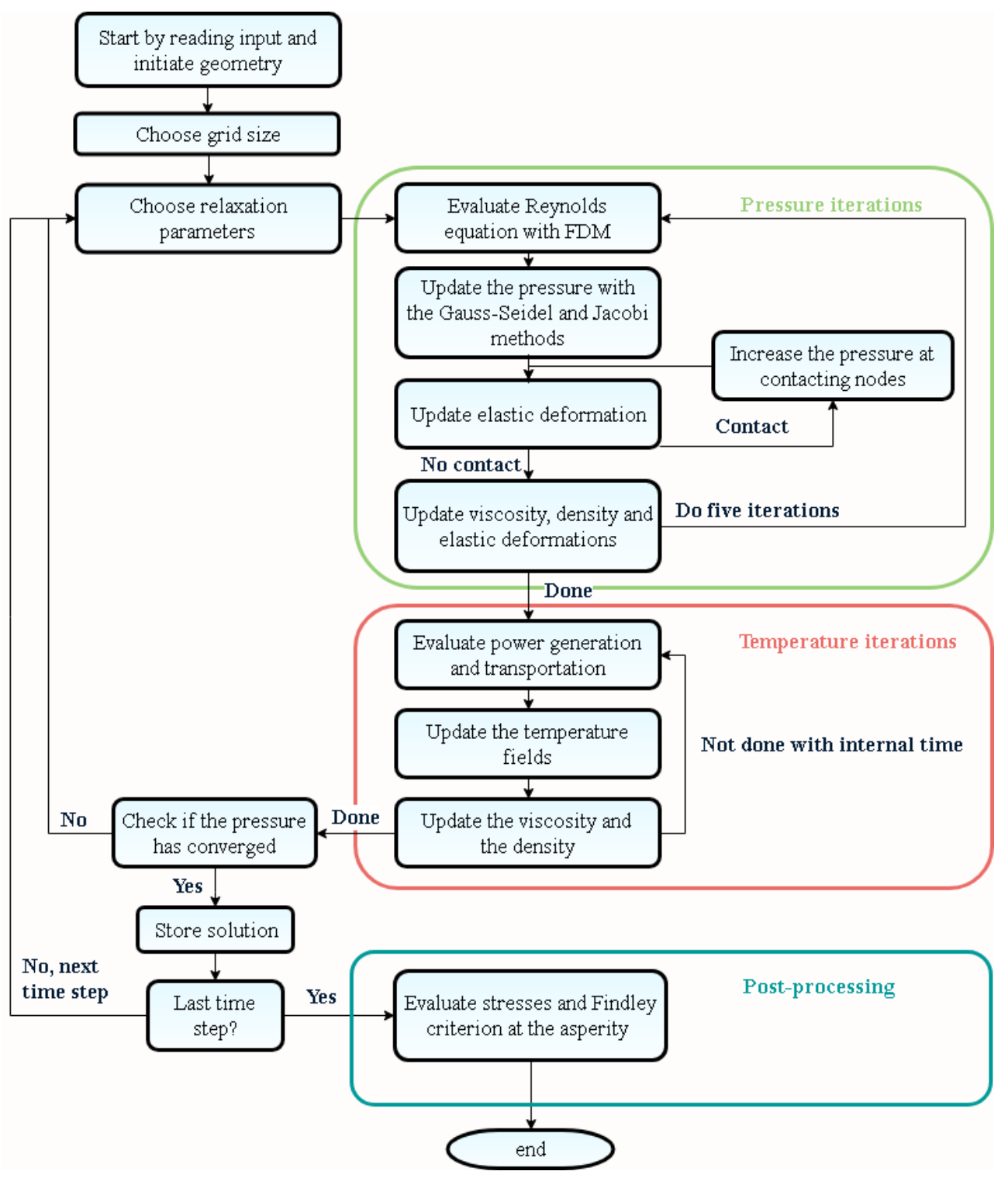

Fig. 2. Overview of the numerical program including couplings between the different physics. 


\section{Results}

The effect of different SRR was investigated through 14 simulations. The speed of the fast surface was $6.3 \mathrm{~m} / \mathrm{s}$ and the speed of the slow surface was adjusted to achieve the desired $S R R$. The asperity was high enough to break through the lubricant and cause metal to metal contact. Other key parameters are presented in Table 1. The lubricant parameters were taken from Larson et al. [5] for the oil named PAO B.

Table 1. Mechanical and numerical parameters including the asperity.

\begin{tabular}{lccc}
\hline Parameter & Symbol & Value & Unit \\
\hline Mean entrainment speed & $u_{\mathrm{m}}$ & $6-6.3$ & $\mathrm{~m} / \mathrm{s}$ \\
Hertzian pressure & $p_{\mathrm{Hertz}}$ & 1.9 & $\mathrm{GPa}$ \\
Equivalent elastic modulus & $E^{\prime}$ & 226 & $\mathrm{GPa}$ \\
Equivalent radius & $r_{x}$ & 10.5 & $\mathrm{~mm}$ \\
Inlet position & $X_{0}$ & 2.0 & - \\
Outlet position & $X_{\mathrm{e}}$ & 1.5 & - \\
Inlet temperature & $\Gamma_{\mathrm{o}}$ & 90 & ${ }^{\circ} \mathrm{C}$ \\
Limit shear stress coefficient & $\gamma$ & 75 & $10^{-3}$ \\
Initial shear stress limit & $\tau_{0}$ & 10 & $\mathrm{MPa}$ \\
Width/wavelength of asperity & $\omega$ & 200 & $\mu \mathrm{m}$ \\
Height of asperity & $\delta$ & 3 & $\mu \mathrm{m}$ \\
Dimensionless material parameter & $G$ & 3.1 & $10^{3}$ \\
Dimensionless speed parameter & $U$ & $4.1-4.3$ & $10^{-11}$ \\
Dimensionless load parameter & $W$ & 4.6 & $10^{-4}$ \\
\hline
\end{tabular}

The key result of the investigation is presented in Fig. 3. It shows that the fatigue risk illustrated by the Findley index is higher for asperity surfaces subjected to negative slip than positive slip. It also shows that pure rolling is the least detrimental case but that fatigue damage is still predicted for the contact conditions in Table 1.

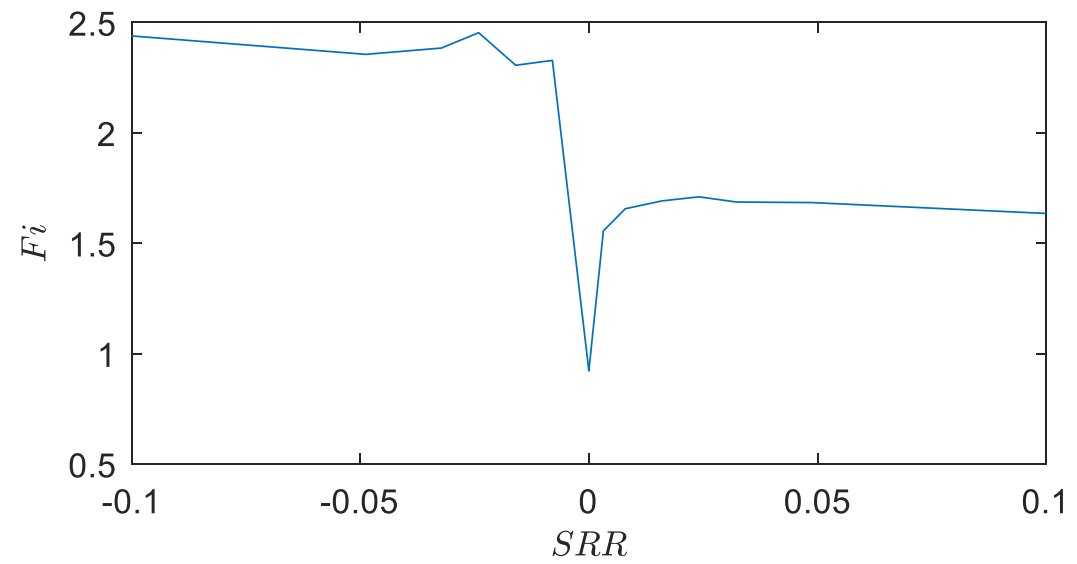

Fig. 3. Maximum Findley index on the asperity as a function of $S R R$.

Fig. 4 illustrates how the Findley index varies in the RD over the asperity. The origin of the $x$-coordinate was placed at the centre of the asperity. This figure shows that the trailing edge, TE, of the asperity is the highest stressed part for negative slip, $S R R<0$. The opposite is true for positive slip where the leading edge, LE, was subjected to the highest stress. 


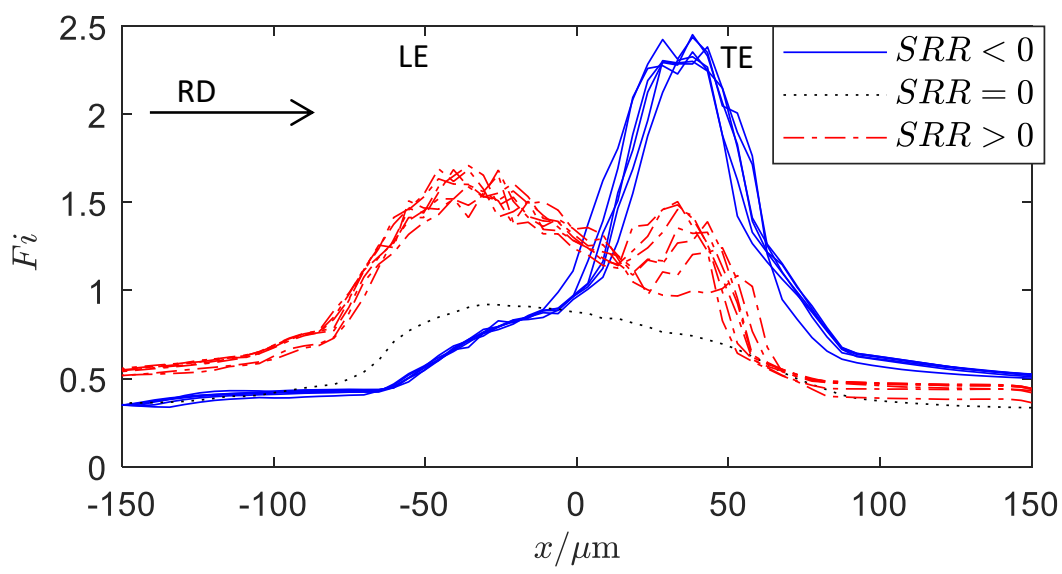

Fig. 4. Findley index as function of the distance $x$ in the RD from the asperity centre.

The Findley index has two components, see Eq. (5). The normal stress and the shear stress amplitude. The surface around the asperity was subjected to high normal stresses when the asperity entered and exited the contact, see Fig. 5. At negative slip the shear tractions increased the normal stress in the inlet compared to that at pure rolling. At positive slip the shear tractions increased the normal stress at the outlet.

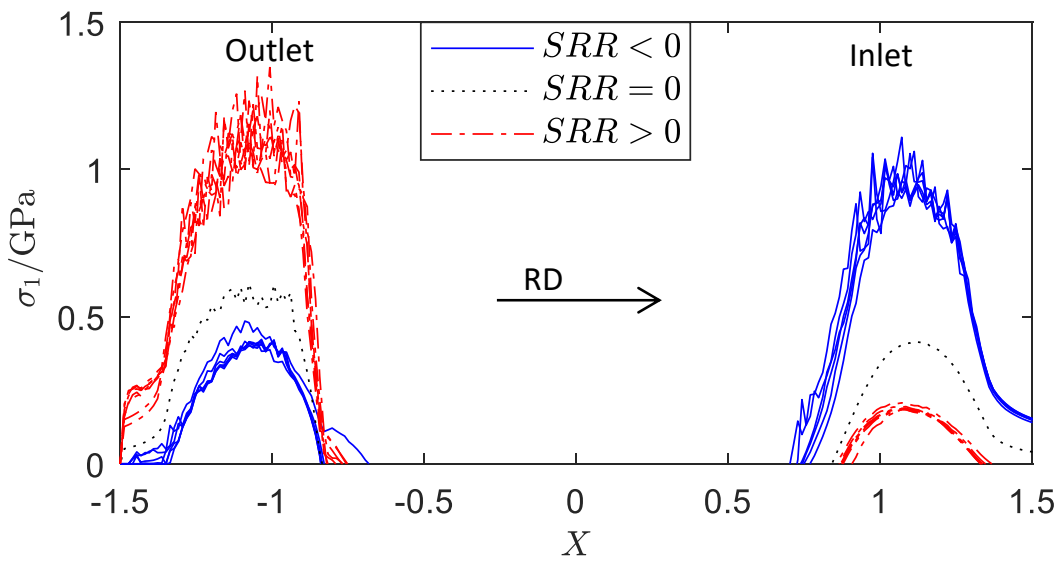

Fig. 5. Maximum of the first principal stress, as function of the dimensionless distance $X$ from the contact centre.

Fig. 6 shows where large $\sigma_{1}$ develop relative to the asperity. When the asperity entered the contact, the tensile stress developed at the trailing edge of the asperity, as is shown in Fig. 1b and Fig 6. When exiting the contact the tensile stress developed on the leading edge of the asperity, see Fig. 6. Figs 5 and 6 show tensile stresses outside the asperity when it enters or leaves the rolling contact. It does not alone explain the $\mathrm{Fi}$ variation in Figs 3 and 4. 


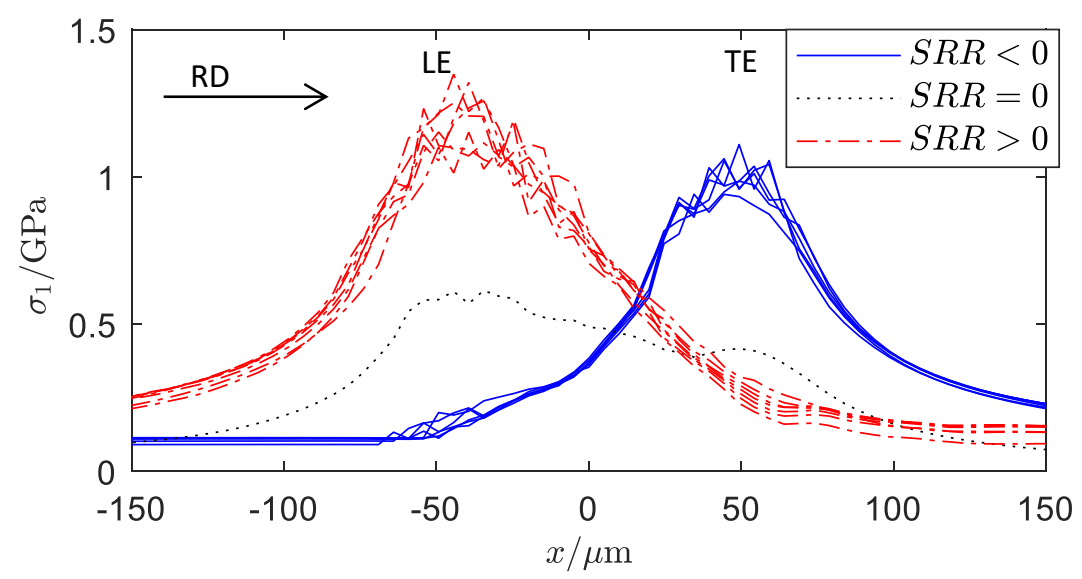

Fig. 6. Maximum of $\sigma_{1}$ as function of $x$ in the RD from the asperity centre.

The position of large shear stresses from metal contact did however explain why the trailing edge was more critically loaded from a fatigue perspective. Fig. 7 shows that the large shear stresses developed only at the trailing edge. Since the $F i$ is a combination of both normal and shear stresses the trailing edge at $S R R<0$ was critical. The reason for the shear stresses being higher at the trailing edge was that metal contact developed close to the trailing edge; see the film height Fig. 8.

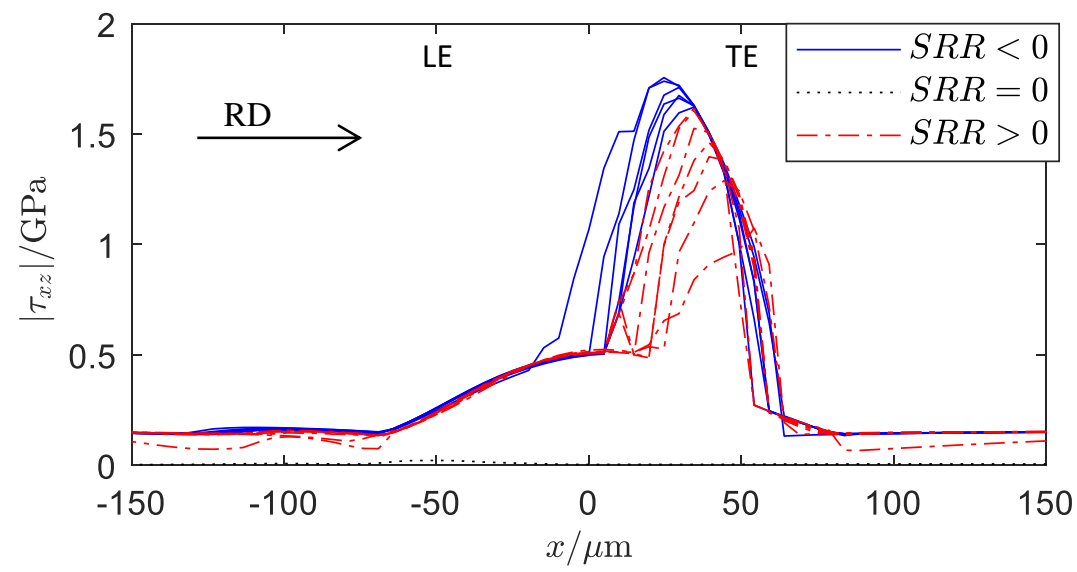

Fig. 7. $\operatorname{Max}\left|\tau_{x z}(x)\right|$ on the asperity surface relative to the asperity centre. 


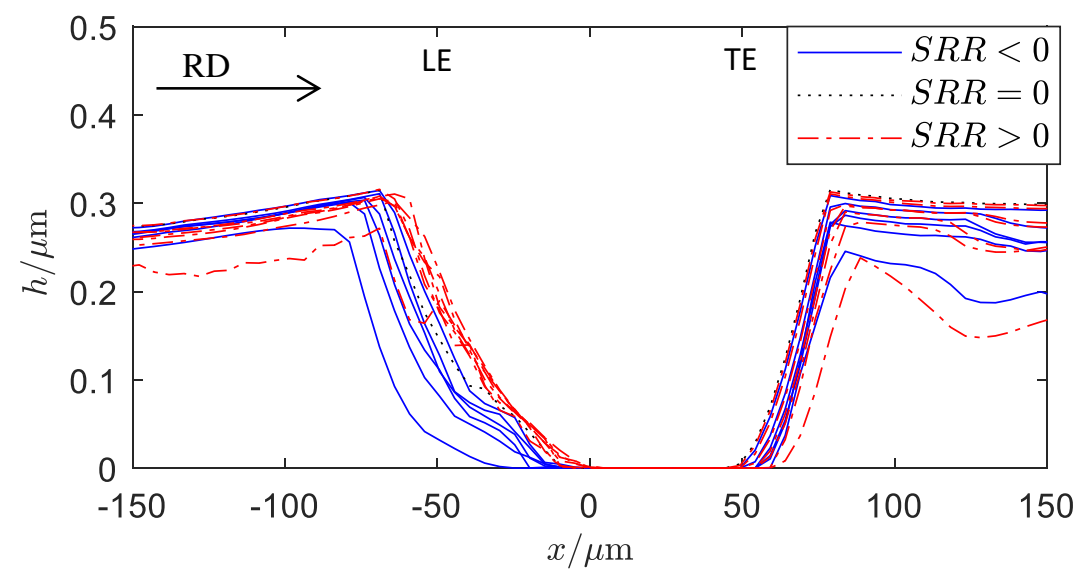

Fig. 8. Minimum film height, as function of the distance $x$ in the RD from the asperity centre. $h=0$ shows that metal contact occurred at the trailing edge of the asperity.

\section{Conclusions}

The contact loads were simulated using thermal elastohydrodynamic lubrication in order to account for the effects of the lubricant. Findley's multiaxial criterion was used and the loads from negative slip were found to be more detrimental than those from positive. The explanation resides in the trailing edge of the asperity breaking through the lubricant as it entered the rolling contact. At negative slip the trailing asperity edge was also the location for the largest tensile stress. At positive slip, on the other hand, the high tensile stress developed at the leading edge, which separated large shear and tensile stresses. Therefore, negative slip is more detrimental than positive slip and pitting is primarily found in surfaces subjected to negative slip. 


\section{References}

1. T.E. Tallian. Failure Atlas for Hertz Contact Machine Elements. ASME Press, New York (1992)

2. B. Alfredsson, M. Olsson. Inclined standing contact fatigue and its relation to surface distress. Proc. of the Eight Int. Fat. Cong., Ed: A.F. Blom, Stockholm June 3-7, 2: 1373-1380 (2002)

3. D. Hannes, B. Alfredsson. Rolling contact fatigue crack path prediction by the asperity point load mechanism. Eng. Frac. Mech., 78: 2848-2869 (2011)

4. C.-M. Everitt, B. Alfredsson. Contact fatigue initiation and tensile surface stresses at a point asperity which passes an elastohydrodynamic contact. Trib. Int., 123 : 234-255 (2018)

5. R. Larsson, P.O. Eriksson, E. Sjöberg, E. Höglund. Lubricant properties for input to hydrodynamic and elastohydrodynamic lubrication analyses. J. Eng. Trib., 214: 17-27 (2000)

6. S. Bair, W.O. Winer. Shear strength measurements of lubricants at high pressure. J. Lub. Tech., 101 : 251-257 (1979)

7. H.S. Cheng, B. Sternlicht. A numerical solution for the pressure, temperature, and film thickness between two infinitely long, lubricated rolling and sliding cylinders, under heavy loads. J. Basic Eng., 87: 695-704 (1965)

8. B. Alfredsson, M. Olsson. Applying multiaxial fatigue criteria to standing contact fatigue. Int. J. Fat., 23 : 533-548 (2001)

9. B. Alfredsson, A. Cadario. A study of fretting friction evolution and fretting fatigue crack initiation for a spherical contact. Int. J. Fat., 26: 1037-1052 (2004)

10. P. Huang. Numerical calculations of lubrication: methods and programs. John Wiley \& Sons, Guangzhou China (2013)

11. C.-M. Everitt, B. Alfredsson. Surface initiation of rolling contact fatigue at asperities considering slip, shear limit and thermal elastohydrodynamic lubrication. Trib. Int., 137: 76-93 (2019) 